\title{
SYSTÈME D'INFORMATION SPATIALE SINUS - INSTRUMENT DE L'ANALYSE GÉOMORPHOLOGIQUE
}

L'article est une tentative d'application du système d'information SINUS au rassemblement, au traitement et à la visualisation de l'information géomorphologique sous forme d'une carte informatisée. SINUS fait partie du groupe de systèmes d'information géographique (Baranowski 1981) basés sur un modèle de trame de données. Cela signifie que les informations sur l'espace sont rassemblées dans des champs unitaires rectangulaires de sorte que le genre donné ou la valeur donnée d'un phénomène caractérise d'une manière équivalente un champ donné.

A titre d'exemple, on a choisi l'aire définie par la feuille d'une carte à l'échelle de 1:25 000 (feuille de Choroszcz). Le terrain en question comprend la courbe de la vallée de la rivière Narew, de Panki à Tykocin, en passant par Zlotoryja.

Le sujet présenté fait appel à l'idée d'élaboration d'une carte géomorphologique à grande échelle abandonnée en raison de ses coûts importants et de la quantité du travail nécessaire. Dans les années 1962-1968 ont été élaborés des systèmes de classement du relief (repris dans les légendes "de Cracovie" et "de Toruń"), sur la base desquels ont été publiées 29 feuilles de cartes géomorphologiques à l'échelle de 1:50 000, élaborées principalement en tenant compte des recherches effectuées sur le terrain.

Du moment de l'élaboration des systèmes de classement cités à l'élaboration d'une carte détaillée des formes du relief, à la lumière des recherches actuelles, plusieurs questions relatives à la reconnaissance géomorphologique devraient être vérifiées. Cela concerne surtout les aires postglaciaires de la plaine de Pologne où l'on abandonne les idées de déglaciation frontale en faveur des idées de glaciation zonale (Bartkowski 1959 b) et superficielle (Musiał 1992, pour le Nord-Est de la Pologne). Il est done nécessaire d'élargir le catalogue de formes postglaciaires et d'envisager les possibilités de les réunir en une banque de données informatiques. Par conséquent, on pourrait actualiser l'information géomorphologique par le biais d'une coopération avec la banque de données.

Une exploitation complète des possibilités de la télédétection dans le 
rassemblement d'informations géomorphologiques est l'un des objectifs de présentation des résultats d'analyse du relief sous forme de carte informatisée. Les recherches sur le terrain ont été réduites aux travaux de vérification du contenu de la carte de photointerprétation, ce qui a permis de diminuer la quantité du travail nécessaire.

La réalisation du problème scientifique ainsi posé comportait quelques étapes, et notamment:

1. Détermination de l'étendue de l'information géomorphologique constituant le contenu de la carte.

2. Choix de matériaux télédétecteurs indispensables à l'obtention d'une information exhaustive concernant la morphographie, la morphométrie et la genèse des formes du relief.

3. Analyse géomorphologique détaillée d'une aire donnée par l'interprétation de photographies aériennes et d'images satellites, en utilisant les cartes thématiques et topographiques disponibles, la littérature scientifique, et en tenant compte des informations obtenues au cours d'une élaboration de carte de revue sur le terrain.

4. Codage des données obtenues par le biais de l'analyse du relief, leurs traitement et visualisation sous forme d'une carte géomorphologique informatisée, élaborée dans le système SINUS. Le système SINUS a été élaboré à l'Institut de Géodésie et de Cartographie, au Centre de Télédétection et d'Information Spatiale par le docteur M. Baranowski.

ad 1. La détermination de l'étendue de l'information géomorphologique incluse dans la carte établie prenait en considération les possibilités de son élaboration continue dans le système de rassemblement d'informations sur l'environnement. Le fait que c'est un système superficiel avec une base de données à organisation de trame signifie que les informations sur l'espace sont rassemblées dans des champs unitaires rectangulaires de sorte qu'un genre donné de phénomène ou la valeur donnée de ce dernier caractérise d'une manière équivalente un champ donné. Autrement dit, toute l'aire concernée est divisée en champs de références spatiales constituant la plus petite unité distinctive de surface à laquelle correspondent les données thématiques. Ce système ne peut donc intégrer les éléments linéaires tels que: fleuves, rebords, encorbellements d'érosion. La carte établie indique uniquement les fleuves pour lesquels il était possible de tracer le lit à l'échelle de 1:25 000. Les autres fleuves qui sont représentés dans la carte sous forme d'une ligne ont été indiqués dans les distinctions: vallées d'eaux de la fonte des glaces à accumulation fluviale dans le fond, fonds de vallées fluviales.

L'information géomorphologique incluse dans la carte élaborée, feuille de Choroszcz, à l'échelle de 1:25 000, est un catalogue des formes de terrain dont la formation est liée à l'accumulation, l'érosion et la destruction des facteurs morphogènes: glacier continental, eaux fluvioglaciaires et glaciolacustres, dénudation, fleuves, vent, facteurs biogéniques et anthropogéniques. Les formes citées dans la légende de la carte (tableau 1) démontrent 
que le relief du terrain concerné s'est formé sous l'effet d'une ablation superficielle du glacier continental provoquant sa décomposition dans la phase finale de déglaciation en blocs de glace morte, sous lintense action d'eaux fluvioglaciaires et, ultérieurement, sous celle d'accumulation éolienne.

Tableau 1

Légende de la carte géomorphologique informatique (1:25 000), feuille de Choroszcz

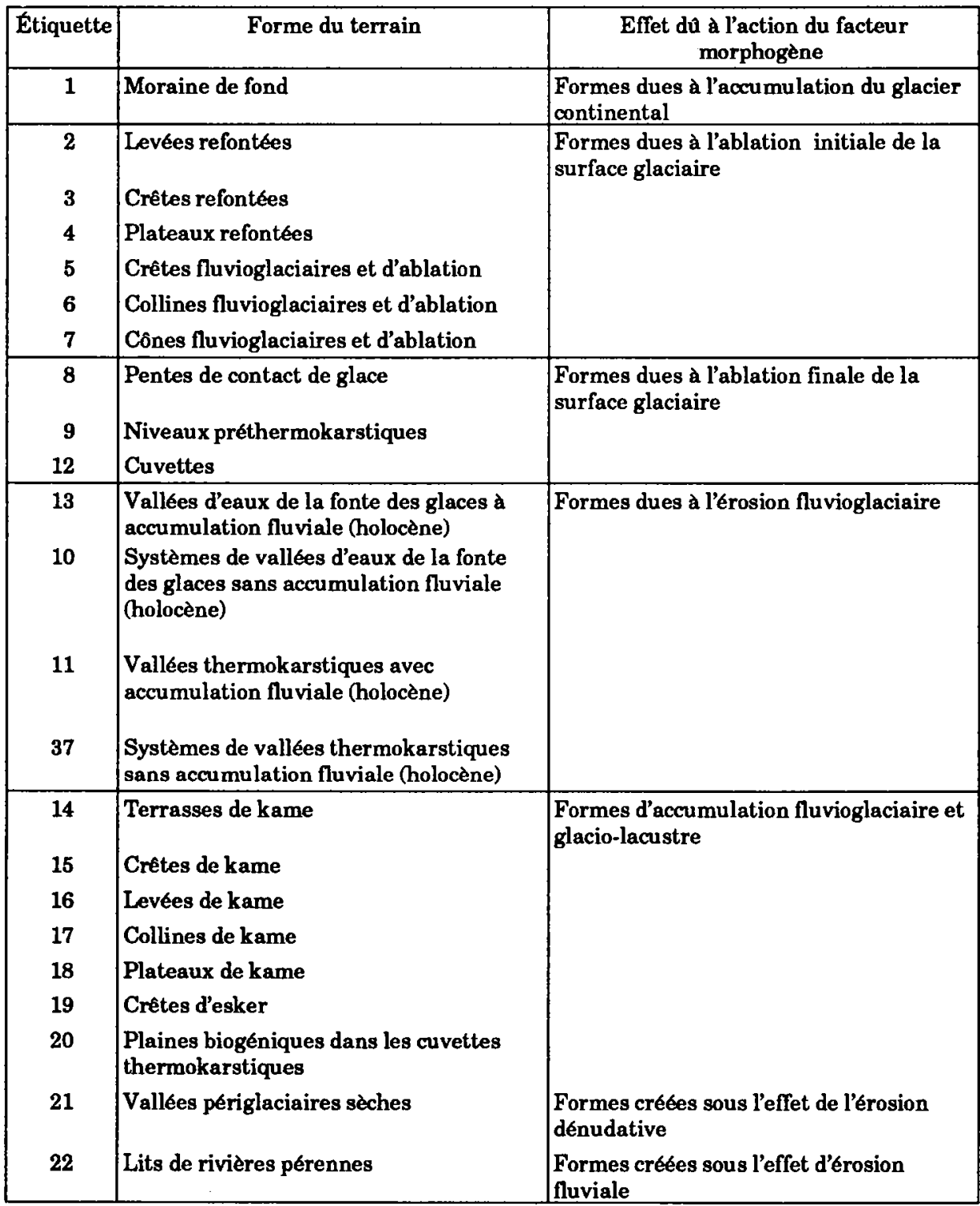




\begin{tabular}{|c|c|c|}
\hline $\begin{array}{l}24 \\
25 \\
40 \\
43\end{array}$ & $\begin{array}{l}\text { Bras morts ou abandonnés secs } \\
\text { Bras morts ou abandonnés avec eau } \\
\text { Ilots de moraine } \\
\text { lles de moraine }\end{array}$ & \\
\hline $\begin{array}{l}26 \\
27 \\
28\end{array}$ & $\begin{array}{l}\text { Fonds de vallées fluviales et de cuvettes } \\
\text { thermokarstiques } \\
\text { Levées naturelles composées de sable } \\
\text { (crétes riveraines) } \\
\text { Cónes alluviaux }\end{array}$ & Formes dues à l'accumulation fluviale \\
\hline $\begin{array}{l}30 \\
31 \\
32 \\
33 \\
23\end{array}$ & $\begin{array}{l}\text { Champs de sable mouvant } \\
\text { Champs de petites dunes irrégulières } \\
\text { Dunes paraboliques } \\
\text { Dunes longitudinales et } \\
\text { crêtes de dunes } \\
\text { Collines de dunes }\end{array}$ & Formes dues à l'érosion \\
\hline $\begin{array}{l}29 \\
36\end{array}$ & $\begin{array}{l}\text { Cuvettes de déflation } \\
\text { Champs de petites cuvettes de déflation }\end{array}$ & Formes d'érosion \\
\hline 34 & $\begin{array}{l}\text { Plaines biogéniques dans les fonds de } \\
\text { vallées et de cuvettes thermokarstiques }\end{array}$ & Formes d'accumulation biogénique \\
\hline $\begin{array}{l}35 \\
38 \\
42 \\
41\end{array}$ & $\begin{array}{l}\text { Sable, gravier, argile } \\
\text { Digues } \\
\text { Fonds d'étangs asséchés } \\
\text { Eaux }\end{array}$ & Formes de destruction et autres \\
\hline
\end{tabular}

ad 2. La complexité des données indispensables à une détermination juste d'unités taxonomiques est l'un des traits essentiels de l'élaboration d'une carte de relief. Les matériaux télédétecteurs constituent une source importante d'informations, toujours insuffisamment exploitée. La carte a été réalisée à partir des photographies aériennes panchromatiques à l'échelle de 1:25 000, prises le 4/10/1987, qui ont permis la distinction, à l'aide du phototone, de la structure et de la texture de l'image, d'aires homogènes du point de vue photomorphique.

ad 3. Ensuite, des analyses morphographiques et morphométriques complètes ont été effectuées à l'intérieur des aires à caractère photomorphique homogène, à l'aide d'une interprétation stéréoscopique détaillée de photographies aériennes. Les analyses ont été complétées de données de la carte topographique. L'image stéréoscopique a permis de déterminer les relations spatiales entre les formes du relief, ce qui a donné l'occasion de formuler des conclusions concernant leur genèse.

L'analyse de la couverture lithologique du terrain étudié et de la constitution interne des formes a été effectuée à partir des cartes de sols et des cartes agricoles à l'échelle de 1:25 000, complétées de recherches de revue sur le terrain. On a rassemblé les caractéristiques morphométriques et lithologiques, et, compte tenu de l'analyse de la structure spatiale des for- 
mes respectives ou de leurs ensembles, un classement génétique des formes du relief a été établi. On a assemblé la carte géomorphologique photointerprétative, feuille de Choroszcz, à l'échelle de 1:25 000 qui, après vérification sur des aires-tests, a été convertie en fond de carte topographique dans le système 65 . Une élaboration cartographique continue a été adaptée à l'analyse dans le système SINUS.

ad 4. L'information analogique incluse dans la carte élaborée a été transformée en information numérique au moyen de la digitalisation par scanner. Pour ce faire, elle a dû être tracée sous forme d'une carte de con-

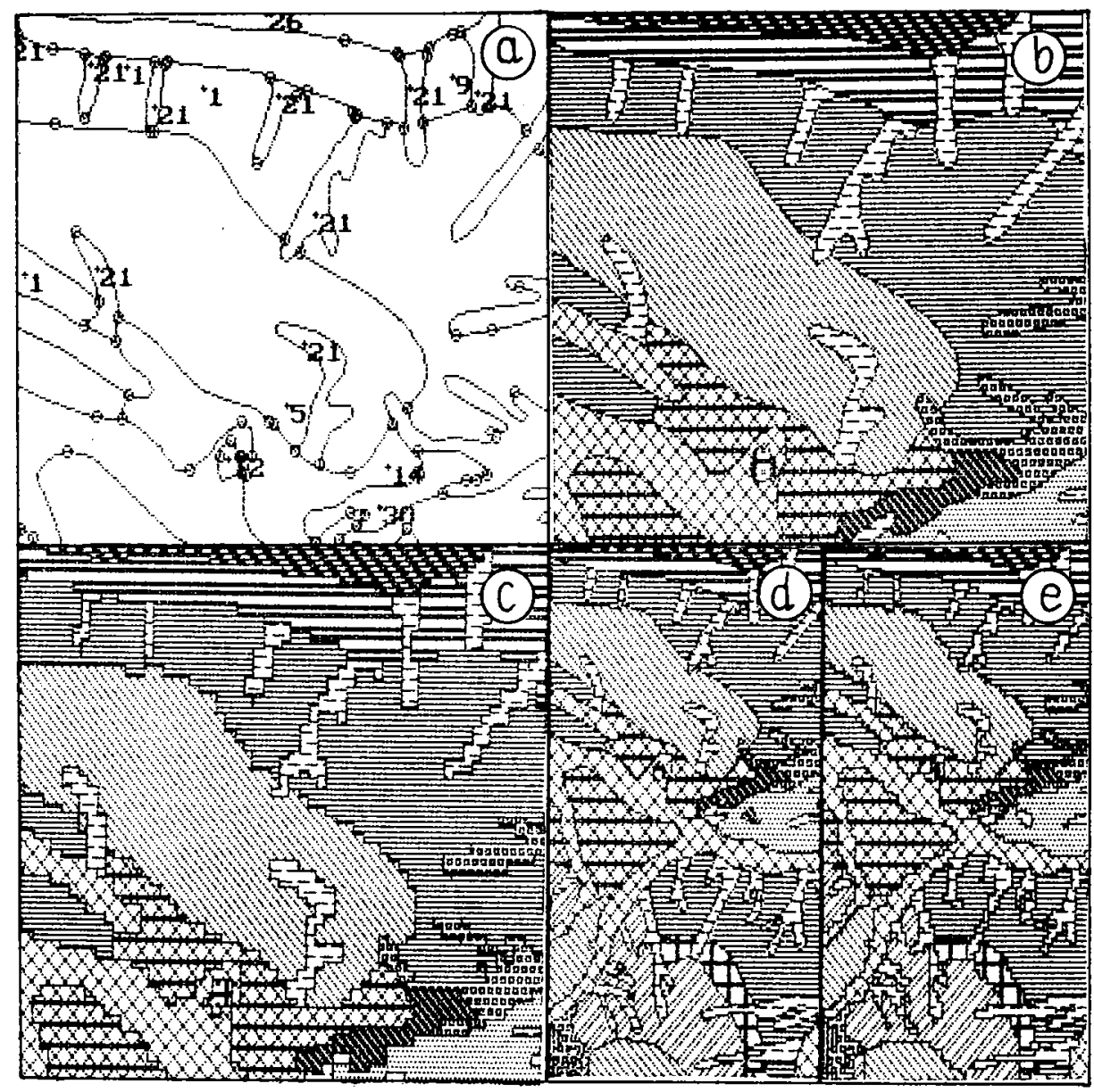

Fig. 1. Étapes d'élaboration d'une carte géomorphologique informatisée (partie Nord-Est de la feuille de Choroszcz, 1:25 000).

a. fragment d'une carte vectorielle (1:25 000). Explications des étiquettes dans le tableau 1 ., b. version monochromatique (1:25 000), c. conversion vectorielle et de trame (1:25 000), d. fragment d'une carte vectorielle en version monochromatique (1:50 000), e. conversion vectorielle et de trame (1: 50000$)$. 


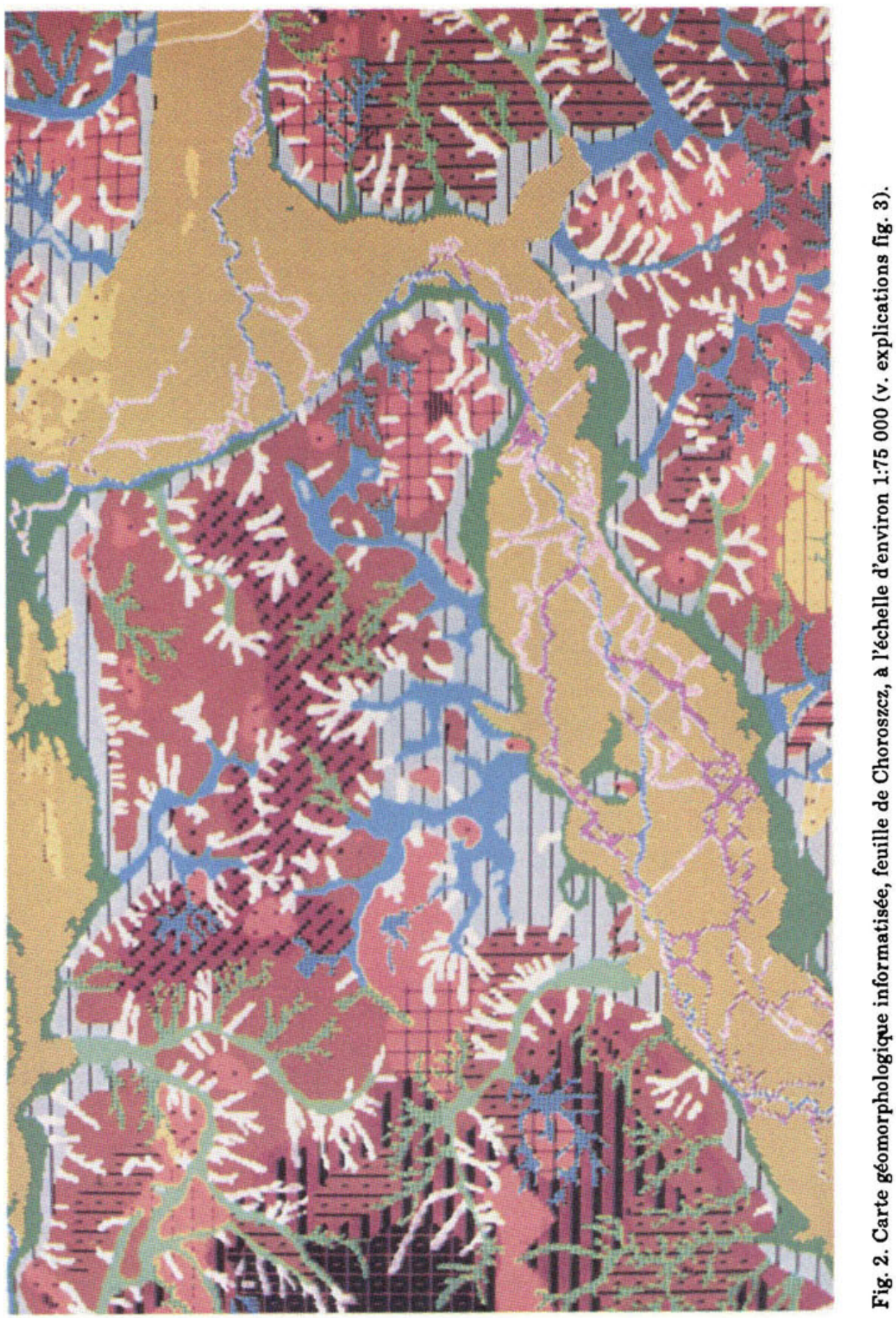




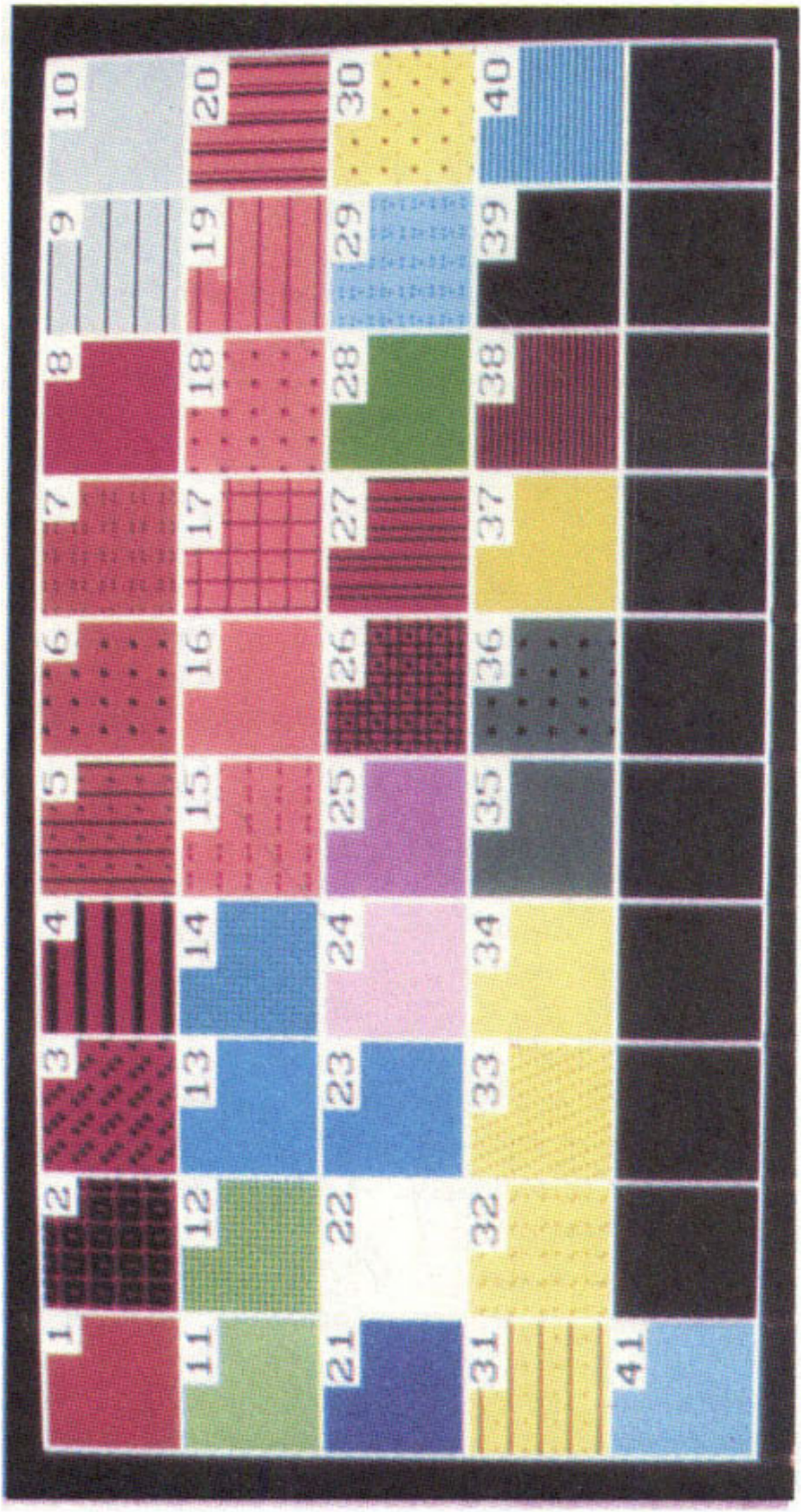

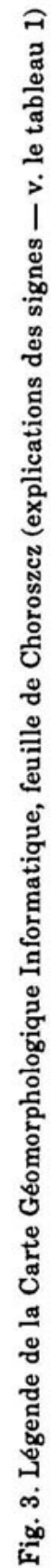


tours dans laquelle les surfaces respectives - unités distinguées du relief - étaient dotées de codes numériques correspondant aux distinctions respectives de la légende.

Dans la technologie utilisée, le retraitement de la carte de contours en forme numérique comportait les étapes suivantes: scannérisation, filtration de l'image, vectorisation de la base de trame, géométrisation et rassemblement des fragments de feuilles.

La scannérisation était le premier processus auquel a été soumise la carte. Il consistait à lire, à l'aide du scanner, un fragment donné de la carte et à décrire l'image sous forme d'une information sur le degré de gris de chaque pixel à l'échelle de 0-255. Ainsi a été créé un ensemble de données de trame représentant une carte de contours. Ensuite, cet ensemble a été soumis à une filtration qui a permis un classement d'informations rassemblées dans une large gamme de gris $(0-255)$ de sorte qu'elles puissent être enregistrées sous forme binaire $(0 ; 1)$. Après, l'ensemble des données de trame sous forme binaire a été converti en ensemble vectoriel. La conversion se produisait en deux étapes. En premier lieu, on a recherché le squelette de la ligne exprimé par une suite d'éléments adjacents à la trame, et ensuite des points de jonction ont été fixés (endroits de convergence de plusieurs lignes). La base vectorielle de données ainsi créée est devenue un matériel approprié pour des travaux continus seulement après une correction géometrique, c'est-à-dire après un retraitement de la base en système 65 . La carte géomorphologique de contours, feuille de Choroszcz, a été scannérisée dans 12 fragments à l'aide desquels, à la suite du processus de jonction, quatre bases de données vectorielles ont été créées.

Toute base vectorielle de données se compose de sept ensembles numériques comprenant les éléments suivants: a) ensemble d'unités superficielles, b) ensemble contenant les adresses des lignes de démarcation des polygones, c) ensemble de lignes, d) ensemble de points, e) ensemble de jonctions, f) ensemble d'étiquettes contenant des codes de distribution géomorphologique présents dans les polygones respectifs, g) ensemble contenant des données sur l'étendue de la base de données. Un polygone est circonscrit par un certain nombre de lignes correspondant au nombre des unités superficielles adjacentes à ce polygone. Chaque ligne se compose de deux jonctions déterminant ses bouts et d'un certain nombre (pris en considération dans la description des lignes) de points intermédiaires. Les jonctions sont des points où convergent au moins trois lignes. Si le polygone est circonscrit par une seule ligne (île, enclave homogène), celle-ci commence et se termine au même point. Dans l'ensemble des points sont conservées les données sur les points intermédiaires entre les jonctions, indiquant la position de la ligne.

L'image scannérisée contient des informations inutiles, créées, par exemple, à la suite d'une circonscription défectueuse de la carte même, que l'on doit supprimer. Il est également possible d'introduire de nouvelles 
données relatives au contenu de la carte, si elles n'avaient pas été prises en considération auparavant. L'image présentant les démarcations des formes, circonscrites sous forme de lignes et de jonctions, et projetée sur l'écran du moniteur est l'effet final de la correction de la base de données, les lignes devant se terminer et commencer au point de jonction. Ensuite ont été introduites les étiquettes déterminant le contenu codé de la carte (fig. 1a). La création d'unités superficielles et l'attribution à l'étiquette d'une couleur concrète ou d'une trame constituaient l'étape suivante. Ainsi a été établie une carte géomorphologique vectorielle en quatre fragments pour la feuille de Choroszcz (1:25 000) qui peut être projetée en couleurs sur l'écran du moniteur ou imprimée en version monochromatique (fig. 1b). Cette carte peut être également obtenue à toutes les échelles, par exemple à l'échelle de 1:50 000 (fig. 1d).

Le groupement de quatre bases de données en une seule, illustrant le contenu de la carte de la feuille entière, exigeait une transition à la forme de trame. La fenêtre analysant le contenu de la carte était de $30 \mathrm{~m}$ sur $30 \mathrm{~m}$, ce qui constitue une distribution spatiale maximum du système SINUS.

Le processus de conversion des structures vectorielles en structures de trame comportait deux étapes. A la première étape - le tracé des lignes de démarcation était dirigé à la grille de trame, à la seconde - les éléments respectifs de la trame ont été remplis d'un code approprié. La première étape a permis de créer une structure vectorielle comportant des éléments linéaires identiques à ceux à l'entrée. Les jonctions et les points ont été rapprochés des points de jonction de la grille de trame, et les lignes déterminant les polygones ont été amenées sur les lignes de la grille de trame de sorte à reproduire la forme originale et le champ des formes délimitées du terrain. La base de trame est donc un tableau à nombre de colonnes et de lignes calculées à partir de données sur l'étendue du territoire de la base. L'étape finale consiste à remplir la base d'un code approprié. Les figures $1 c$ et $1 e$ illustrent un exemple de conversion vectorielle et de trame pour les échelles de 1:25 000 et 1:50 000. Il est également possible de projeter la carte géomorphologique en entier sur l'écran du moniteur sous forme d'une légende de couleur ou de l'introduire dans le ploter, imprimante en version monochromatique, (fig. 2,3 ). Le contenu de la carte pour la feuille de Choroszcz à l'échelle de 1:25 000, enregistré sous forme numérique, constitue la première tentative en Pologne de créer une banque de données rassemblant des informations géomorphologiques localisées au niveau spatial. Grâce à l'utilisation d'une conversion vectorielle et de trame, qui est indispensable aux procédures d'interférence de différentes couches d'informations, le contenu de la carte est une base pour des recherches sur les corrélations avec d'autres éléments de l'environnement naturel. 


\section{BIBLIOGRAPHIE}

Baranowski M., 1989, „System informacji o uksztaltowaniu środowiska SINUS" (Système d'information sur la formation de l'environnement SINUS), dans: Monitoring zmian zagospodarowania przestrzennego, Konferencja Problemowa TUP, Warszawa. (Monitorage des changements d'aménagement de l'espace, Conférence TUP).

Bartkowski T., 1969, „Deglacjacja strefowa deglacjacja normalna na obszarach niżowych (na wybranych przykładach Polski Zachodniej i Pöłnocnej)", Bad. Fizjogr. nad Polskq Zach., Seria A., Geogr. Fiz., t.23. (Déglaciation zonale - déglaciation normale dans les plaines - sur les exemples choisis de l'Ouest et du Nord de la Pologne).

M usi ał A., 1992, Studium rzeźby glacjalnej pótnocnego Podlasia, Rozprawy UW, wyd. UW, Warszawa. (Étude du relief glaciaire du Nord de la région de Podlasie, Dissertations de l'Université de Varsovie. 